Two-dimensional Forster resonance energy transfer (2-D FRET) and the membrane raft hypothesis

Peer-reviewed author version

ACASANDREI, Maria; DALE, Robert; VAN DE VEN, Martin \& AMELOOT, Marcel (2006) Two-dimensional Forster resonance energy transfer (2-D FRET) and the membrane raft hypothesis. In: CHEMICAL PHYSICS LETTERS, 419(4-6). p. 469-473.

DOI: 10.1016/j.cplett.2005.11.104

Handle: http://hdl.handle.net/1942/1682 


\title{
Two-dimensional Förster resonance energy transfer (2-D FRET) and the membrane raft hypothesis.
}

\author{
Maria A. Acasandrei, ${ }^{\mathrm{a}, \mathrm{b}}$ Robert E. Dale, ${ }^{\mathrm{b}, \mathrm{c}}$ Martin vandeVen, ${ }^{\mathrm{b}}$ and Marcel Ameloot ${ }^{\mathrm{b}}$ \\ ${ }^{a}$ Health and Environmental Physics Department, \\ "Horia Hulubei” National Institute for Physics and Nuclear Engineering, \\ PO Box MG-6, Bucharest Magurele, \\ R-76900, Romania \\ ${ }^{\mathrm{b}}$ Hasselt University and transnational University Limburg, School of Life Sciences, \\ Biomedical Research Institute, Agoralaan, \\ BE-3590 Diepenbeek, Belgium
'King's College London, School of Biomedical and Health Sciences, Randall Division for Cell and Molecular Biophysics, $3^{\text {rd }}$ Floor, New Hunt's House, Guy's Hospital Campus, London SE1 1UL, United Kingdom

\section{Address for correspondence:}

\author{
M. Ameloot \\ Hasselt University \\ Biomedical Research Institute \\ Agoralaan, Building D \\ BE-3590 Diepenbeek \\ Belgium
}

FAX: 32-11-268-599

e-mail: marcel.ameloot@uhasselt.be

A preliminary account of this work was presented in poster form at the 2004 Biophysical Society Discussion Meeting: “Probing Membrane Microdomains”, October 28-31, 2004 Asilomar, California, USA [1].

Keywords: FRET, 2-dimensional, resonance energy transfer, cell plasma membrane, lipid raft, microdomain

\section{Running title: FRET and membrane rafts}




\begin{abstract}
A model for analyzing Förster resonance energy transfer (FRET) data in relation to the cell plasma membrane raft hypothesis is developed to take into account (a) the distribution of FRET donors and acceptors at the surface of probing antibody fragments specific for a putative raft component, (b) partitioning of the raft component between raft and non-raft areas of the membrane, and (c) the dependence of the raft partition on the expression level of the considered component. Analysis of relevant FRET data in the literature according to this model provides support for the raft hypothesis.
\end{abstract}

\title{
Introduction
}

Lipid rafts in cellular membranes are clusters enriched in cholesterol and sphingolipids into which certain proteins partition preferentially (see, e.g., [1-8]). The operational definition of lipid rafts is based on the differential solubility of membrane lipids and proteins in detergents (see, e.g., [9]). Since application of a detergent can alter the phase behavior of membranes [10], alternative in situ methods are required to support these results. Among these, Förster resonance energy transfer (FRET) [11, 12] provides a promising approach as this phenomenon is sensitive to separations of between $\sim 1$ to $10 \mathrm{~nm}$ (see, e.g., $[13,14])$.

Extensive treatment of the case of FRET for 2-D systems is to be found in the literature, both in the strict point-dipole interaction limit (e.g., $[15,16])$, and for various excluded area and/or volume scenarios (e.g., [16-19]). In recent years, possible localization of functional proteins into rafts in cell plasma membranes has been investigated by FRET between both unlike [see 4, and, e.g., 20-29] and like [28, 30] donors and acceptors. However, as far as the authors are aware, no theory so far presented in the literature accounts in detail for the most common experimental case of expected surface distributions of the donors and acceptors on separately D- and A-labeled probing proteins. 
A numerical integral solution for a spherical, randomly surface-labeled protein model is presented here, and applied to a literature example [20] of putative cell-membrane raft proteins specifically probed with separately surface-labeled D- and A-bearing antibody fragments (Fab), to test whether or not the reported FRET data might be consistent with the presence of rafts.

\section{Theoretical basis}

The efficiency $E$ of FRET for a random planar two-dimensional distribution of A about $\mathrm{D}$ in the point-dipole interaction limit, is defined by:

$$
E=1-\left(1 / \tau_{0 D}\right) \int_{t=0}^{\infty} \exp \left[-\left(t / \tau_{0 D}\right)\right] \exp \left[-c_{A} \int_{R=0}^{\infty} 2 \pi R\left(1-\exp \left[-\left(1 / \tau_{0 D}\right)\left(R_{0} / R\right)^{6}\right]\right) d R\right] d t
$$

where $t$ denotes the time after $\delta$-pulse excitation of $\mathrm{D}, \tau_{0 D}$ is the decay time of $\mathrm{D}$ in absence of A, $c_{A}$ the number concentration of acceptors per unit area, $R$ the separation of $\mathrm{A}$ from $\mathrm{D}$, and $R_{0}$ the Förster separation for an isolated pair of $\mathrm{D}$ and $\mathrm{A}$ which leads to an efficiency of $50 \%$.

\section{INSERT Figure 1 HERE}

Figure 1 depicts a single pair of separately D and A surface-labeled identical spheres in a population randomly distributed over a plane. The plane corresponds, in the present context, either to the plane of a raft or to that of an area outside rafts. For such a population, the energy transfer efficiency from donors to acceptors when the area around the donor-bearing protein is effectively infinite (e.g., in rafts for raft dimensions $>>R_{0}$, so that edge effects are negligible) is given by:

$$
E\left(c_{A}\right)=1-\left(1 / \tau_{0 D}\right) \int_{t=0}^{\infty}\left[\exp \left(-\left[t / \tau_{0 D}\right]\right) \times \frac{1}{2} \int_{\psi=0}^{\pi} \exp \left(-c_{A} \frac{1}{2} \int_{R=2}^{\infty} \int_{r=0}^{\pi} 2 \pi R \mathrm{~J}(\alpha, R, \psi, t) d R \sin \alpha d \alpha\right) \sin \psi d \psi\right] d t
$$


where the integration variables $\alpha, R$, and $\psi$ are defined as in Figure $1, r$ is the radius of the labeled spheres, and $c_{A}$ now the effective (pseudo-)2-dimensional number concentration of acceptor molecules per unit area, equal to that of the spheres themselves multiplied by the labeling ratio (average number of acceptors per sphere). $\mathrm{J}(\alpha, R, \psi, t)$ is given by: $\mathrm{J}(\alpha, R, \psi, t)=1-\exp \left(-\left[t / \tau_{0 D}\right] R_{0}^{6}\left[R^{2}+2 r^{2}+2 r\left(R \cos \psi+\left[R^{2}+r^{2}+2 R r \cos \psi\right]^{1 / 2} \cos \alpha\right)\right]^{-3}\right)$

\section{INSERT Figure 2 HERE}

The FRET efficiency given by Eq. (2), presented as a function of $c_{A}$ normalized to units of number concentration per $R_{0}^{2}$ for sphere radii $r=R_{0} / 2$ appropriate to the case in hand (see below), is contrasted in Figure 2 with that predicted by the simple D-A case of Eq. (1). The large differences in the courses and rates of change of $E$ with $c_{A}$ are due to the relatively large excluded volumes of the spheres from and into which transfer cannot occur.

A simple model of partitioning of the raft-specific "marker" protein $P$ into raft areas making up an overall fraction $f_{R}$ of the cell surface area, the individual raft areas themselves being distributed randomly over the cell membrane surface, is applied. Specifically D- and A-labeled anti- $P$ Fab fragments monitor the partitioning of $P$ between raft and non-raft regions according to their overall concentrations (expression level in the case of $P$ ) in the membrane, and their common partition coefficient $K_{p}$ :

$$
K_{p}=c_{D R} / c_{D \bar{R}}=c_{A R} / c_{A \bar{R}}=c_{P R} / c_{P \bar{R}}
$$

where $c_{D R}, c_{A R}, c_{P R}$ are the donor and acceptor fluorophore, and raft-specific component $P$ number concentrations, respectively, within raft areas, and $c_{D \bar{R}}, c_{A \bar{R}}, c_{P \bar{R}}$ those outside the raft areas. The observed FRET efficiency is the average of that for donors within individual 
raft areas, $E_{\text {raft }}$, and donors outside the rafts, $E_{\overline{r a f t}}$, weighted by the fraction of donors in each situation: $f_{D R}$ and $f_{D \bar{R}}=1-f_{D R}$, respectively:

$$
E\left(c_{A T}\right)=f_{D R} E_{\text {raft }}\left(c_{A R}\right)+\left(1-f_{D R}\right) E_{\overline{r a f t}}\left(c_{A T}\right)
$$

where, $c_{A T}$ is the effective average number concentration of acceptor fluorophores per unit area of total membrane, which is appropriate for transfer from donors situated outside raft areas to all acceptors, both without and within raft areas, while $c_{A R}$ is appropriate for transfer from donors within the raft areas in the absence of edge effects (these effects, which become significant when the raft dimensions approach $R_{0}$, do not qualitatively affect the analysis and conclusions here, and will be discussed elsewhere). The latter concentration is given by:

$$
c_{A R}=\frac{K_{p}}{1+f_{R}\left(K_{p}-1\right)} c_{A T},
$$

while the fraction $f_{D R}$ of donor (as also equivalently the fraction of acceptor $\mathrm{A}$, fraction of total antibody fragment, and fraction of antigen $P$ ) in raft areas is given by:

$$
f_{D R}=\frac{f_{R} K_{p}}{1+f_{R}\left(K_{p}-1\right)}
$$

The overall fractional area taken up by rafts depends on the expression level of the raftspecific component $P$ of interest, and is taken to be given by a simple saturation function:

$$
f_{R}=\frac{K c_{P}}{1+K c_{P}}
$$

where $K$ is the equilibrium constant, and $c_{P}$ is the surface concentration of $P$, given by:

$$
c_{P}=\frac{c_{A T}}{s_{A} S}\left(\frac{1+\rho_{A: D}}{\rho_{A: D}}\right)
$$

in which $s_{A}$ is the (average) acceptor labeling ratio per Fab molecule, $\rho_{A: D}$ the ratio of Alabeled to D-labeled Fab, and $S$ the degree of saturation of the antigenic sites. 


\section{Application and Discussion}

The above theoretical treatment is applied here to data reported in the literature ([20], Figure 4b: FRET efficiency $v s$. acceptor intensity for $\rho_{A: D}=3$ ), and kindly made available to the authors, together with a calibration factor of $6.0 \times 10^{-5}$ per $R_{0}^{2}$ for the number concentration of acceptor in terms of acceptor intensity. These data were obtained by imaging FRET microscopy, and relate to the distribution of the glycosylphosphatidylinositol-anchored protein 5'-nucleotidase (GPI-anchored 5'NT), a putatively raft-associated enzyme, in the outer half of the apical bilayer plasma membrane of a transfected cultured Madin-Darby canine kidney cell line (MDCK Type II). The Fab fragments specific for the protein were labeled separately via their surface lysine $\varepsilon$-amino groups with the fluorescent cyanine dyes Cy3 (donor) and Cy5 (acceptor, labeling ratio $s_{A}=4.1$ ). These dyes form a FRET pair having a Förster $R_{0}$ taken to be $5 \mathrm{~nm}$ [20]. 3:1 mixtures of the A-labeled to D-labeled Fab preparations were applied at saturating concentrations ( $S=1$ ) of $200 \mu \mathrm{g} / \mathrm{mL}$ total Fab to the cells on a cover slip, and the cells subsequently fixed with $4 \%$ formaldehyde. The overall concentrations of acceptor in the selected membrane regions of interest were monitored by the fluorescence intensity of directly excited acceptor Cy5. The FRET efficiency averaged over a small single selected square patch of apical plasma membrane per cell was determined on each of 72 separate cells, using the donor bleaching method as described in [20]. On the basis of their modeling, Kenworthy and Edidin [20] concluded that a small fraction of rafts, maybe up to $25 \%$ of the total surface area, would not have been detected, and that simple clustering of small numbers, for example dimerization, of the specific molecules would explain their findings However, it may be noted that the measured data (reproduced here in Figure 3, below) appear to level off at quite a low FRET efficiency (here around 25\% or so), a feature strongly qualitatively shared with virtually all the rest of the data displayed in [20]. On the 
other hand, their dimer simulations presented in Reference 20, Figure 5 (actually relating to a different, albeit similar, data set), for example, indicate a continuing increase in FRET efficiency with concentration at the overall levels observed. This leveling off is, at least qualitatively, consistent with the presence of a two-component system, of raft and non-raft regions, each having random distributions of the antigen within them, but at higher concentrations within raft areas than outside. Using the above theoretical treatment, the consistency of such a raft model with the data was checked.

Making use of the calibration factor supplied, but adjusted for projected area effects (see below), these data were fitted for $K$ and $K_{p}$ according to Eqs. (5)-(9), together with efficiencies given by a slightly modified form of Eq. (2), introduced to take account of a low level of background FRET (to other, naturally occurring, acceptors in and/or close to the membrane) found experimentally in this case (efficiency 2.5\%). The effect was approximated as a simple competing process shortening the lifetime of the donor in absence of the specific added acceptor, by entering in the first exponent in Eq. (2) the lifetime that would give rise to this effect instead of the lifetime $\tau_{0 D}$ of the donor in absence of any acceptor. The analysis was carried out by unweighted non-linear least squares fitting of the theoretical $E\left(c_{A}\right)$ relationship to the acceptor intensity data using the Globals Unlimited ${ }^{\mathrm{TM}}$ (Laboratory for Fluorescence Dynamics, Urbana, Illinois, USA) software package. In order to minimize the complexity, and thereby insupportable inefficiency, of a non-linear least squares analysis of the experimental data using the multiply-integrating theoretical relationship directly, a simpler relationship was substituted and employed for the analysis. The form chosen was multi-exponential in the acceptor concentration. Its parameters (preexponentials and multipliers of the acceptor concentration in the exponents) were obtained by fitting to the theoretical course of FRET efficiency given by Eq. (2) modified as above for background FRET, using the "Find" function in MathCad 2001i (MathSoft, Cambridge, 
Massachusetts, USA). A sum of seven exponentials was found to be required to fit the theoretical function to a tolerance of better than $10^{-4}$ across the concentration range displayed in Figure 2.

The intensity to number concentration calibration factor supplied by the authors (see above) was not used directly, as it assumed an effective cell surface area equal to the projected area under the microscope. Visualization of MDCK Type II cell plasma membrane surfaces show the presence of many microvilli, approximately cylindrical protrusions. Estimates of about $20-22 \%$ for the fractional surface area taken up by microvilli were made from micrographs of freeze-fractured monolayer-cultured cells [31, 32], i.e., a density of $\sim 13-14$ per $\mu \mathrm{m}^{2}$ and radius of about $70 \mathrm{~nm}[31,32]$. Other estimates made of the latter from the literature were $\sim 80 \mathrm{~nm}$ [33], and $\sim 60 \mathrm{~nm}$ [34]. Microvillus lengths are much less certain, varying from an average of $\sim 0.2 \mu \mathrm{m}$ [35], through estimates made from the literature of $\sim 0.5 \mu \mathrm{m}$ [34] to up to between $\sim 1$ and $2 \mu \mathrm{m}$ [33]. Here, “representative” values of 13 per $\mu \mathrm{m}^{2}, 0.07 \mu \mathrm{m}$, and $1 \mu \mathrm{m}$ are taken for the density, radius and length, respectively, leading to an overall true-to-projected area ratio of about 6.7, and thus a "representative” calibration factor close to $9 \times 10^{-6}$ per $R_{0}^{2}$ (i.e., per $25 \mathrm{~nm}^{2}$ ). This value was used in the analysis, as were also values 3-fold larger and smaller to obtain some estimate of the tolerances of the derived parameters.

\section{INSERT Figure 3 HERE}

Excellent fits to the data were obtained in each case, as can be seen in Figure 3. The small initial lag in the onset of FRET efficiency with concentration is caused by the concentration-dependence of raft formation in the model applied. For the calibration factors $\left(3 \times 10^{-6}, 9 \times 10^{-6}, 27 \times 10^{-6}\right)$ per $R_{0}^{2}$, the fitted values were $K \sim(14.4,16.6,27.2) R_{0}^{2}$, or (75, 225, 675) $\times 10^{-6} \mu \mathrm{m}^{2}$, and $K_{p} \sim(169,55,18)$, respectively. From these, using Eqs. (6)- 
(9), maximum number concentrations of $\sim(6.86,6.96,7.27) \times 10^{-2}$ per $R_{0}^{2}$, or $\sim(2.72,2.75$, 3.01) $\times 10^{3}$ per $\mu \mathrm{m}^{2}$, were obtained for the total antigenic protein in the putative rafts, together with maximum fractions of surface area taken up by raft of $f_{R}^{\max } \sim(0.10,0.20$, 0.38), and maximum fractions of antigen in raft areas of $\left(f_{R} K_{p} /\left[1+f_{R}\left(K_{p}-1\right)\right]\right)^{\max } \sim(0.925,0.931,0.944)$. The maximum number concentrations of total Fab in raft areas are very similar for all three calibration factors. They are more than an order of magnitude smaller than that corresponding to its close hexagonal packing limit of just over one molecule per $R_{0}^{2}(2 / \sqrt{3}$, or $\sim 1.154)$ and thus appear to be physically reasonable.

\section{Conclusions}

In the above, a simplified quantitative raft model is described and used to interpret FRET data for D- and A-labeled Fab bound to cell plasma membrane “marker” proteins putatively partitioned into rafts, modeling raft formation as dependent on their expression level. As the basis for analysis according to this model, theoretical FRET efficiencies as a function of effective acceptor concentration were derived for donors and acceptors considered as point dipoles randomly separately distributed on the surfaces of spherical antibody fragments bound specifically to the raft "marker” antigens in locally 2-D random distributions within and outside raft areas.

Despite the effect of uncertainties in estimation of true-to-projected overall cell surface areas, it is shown that the model of raft formation applied in this study is not incommensurate with the data taken here as a paradigm for the FRET investigations reported by Kenworthy and Edidin [20], and lends quantitative support to the raft hypothesis, at least for the apical plasma membranes of the transfected MDCK II cells used in those investigations. While the existence of rafts in the membranes of these cells is not 
thereby unequivocally proved, the Kenworthy-Edidin FRET results are inferred to be consistent with at least the simple model of raft formation invoked here. Ongoing work comparing this with other raft models as well with specific dimer ones for all the Fab data provided, including that for different ratios of donor-labeled to acceptor-labeled Fabs, will be reported at length elsewhere.

\section{Acknowledgements}

The authors gratefully acknowledge the free receipt of relevant original data, as well as an intensity-concentration calibration factor for it, from Professors Anne Kenworthy and Michael Edidin [20]. MAA gratefully acknowledges the support of the Bilateral Project betweeen Flanders and Romania for her stay at the Universiteit Hasselt during part of this work. RED likewise thanks the Universiteit Hasselt for partial support during a number of visits. MvdV and MA acknowledge support from the "tUL-impulsfinanciering".

\section{References}

[1] A.K. Kenworthy, Traffic 6 (2005) 518.

[2] K. Simons, E. Ikonen, Nature. 387 (1997) 569.

[3] M. Edidin, Curr. Opin. Struct. Biol. 7 (1997) 528.

[4] M. Edidin, Annu. Rev. Biophys. Biomol. Struct. 32 (2003) 257.

[5] L.J. Pike, J. Lipid Res. 44 (2003) 655.

[6] S. Mayor, M. Rao, Traffic 5 (2004) 231

[7] S. Mukherjee, F.R. Maxfield, Annu. Rev. Cell Dev. Biol. 20 (2004) 839.

[8] B.C. Lagerholm, G.E. Weinreb, K. Jacobson, N.L. Thompson, Annu. Rev. Phys. Chem. 56 (2005) 309.

[9] H. Shogomori, D.A. Brown, Biol. Chem. 384 (2003) 1259.

[10] H. Heerklotz, Biophys. J. 83 (2002) 2693.

[11] Th. Förster, Ann. Physik 2 (1948) 55. 
[12] Th. Förster, Zeits. Naturforsch. 4a (1949) 321.

[13] R.M. Clegg, Curr. Opin. Biotechnol. 6 (1995) 103.

[14] R.M. Clegg, in: X. F. Wang, B. Herman (Eds.), Fluorescence Imaging Spectroscopy and Microscopy, Wiley, New York, 1996, p. 179.

[15] A.G. Tweet, W.D. Bellamy, G.L. Gaines, Jr., J. Chem. Phys. 41 (1964) 2068.

[16] P.K. Wolber, B.S. Hudson, Biophys. J. 28 (1979) 197.

[17] B.K.-K. Fung, L. Stryer, Biochemistry 24 (1978) 5241.

[18] B. Snyder, E. Freire, Biophys. J. 40 (1982) 137.

[19] J. Yguerabide, Biophys. J. 66 (1994) 683.

[20] A.K. Kenworthy, M. Edidin, J. Cell Biol. 142 (1998) 69.

[21] A.K. Kenworthy, M. Edidin, Methods Mol. Biol. 116 (1999) 37.

[22] D.A. Zacharias, J.D. Violin, A.C. Newton, R.Y. Tsien, Science 296 (2002) 913.

[23] B.J. Nichols, Current Biology 13 (2003) 686.

[24] J.R. Silvius, Biophys. J. 85 (2003) 1034.

[25] H. Wallrabe, M. Elangovan, A. Burchard, A. Periasamy, M. Barroso, Biophys. J. 85 (2003) 559.

[26] C. Zurzolo, G. van Meer, S. Mayor, EMBO Reports 4 (2003) 1117.

[27] I. Gombos, C. Detre, G. Vamosi, J. Matko, Immunol. Lett. 92 (2004) 117.

[28] P. Sharma, R. Varma, R.C. Sarasij, Ira, K. Gousset, G. Krishnamoorthy, M Rao, S. Mayor, Cell 116 (2004) 577.

[29] R.F.M. de Almeida, L.M.S. Loura, A. Fedorov, M. Prieto, J. Mol. Biol. 346 (2005) 1109.

[30] R. Varma, S. Mayor, Nature 394 (1998) 798.

[31] M. Cereijido, J. Ehrenfeld, I. Meza, A.. Martinez-Palomo, J. Membrane Biol. 52 (1980) 147. 
[32] J.H. Todd, D.A. Sens, M.A. Sens, D. Hazen-Martin, Microsc. Res. Technique 22 (1992) 301.

[33] C. Butor, J. Davoust, Exp. Cell Res. 203 (1992) 115.

[34] S.A. Francis, J.M. Kelly, J. McCormack, R.A. Rogers, J. Lai, E.E. Schneeberger, R.D. Lynch, Eur. J. Cell Biol. 78 (1999) 473.

[35] K. Poole, D. Meder, K. Simons, D. Müller, FEBS Lett. 565 (2004) 53. 


\section{Figure Captions}

Figure 1. Geometrical relationships of arbitrarily placed FRET donor (D) and acceptor (A) moieties on the surfaces of separate spheres of radius $r$ lying on a plane at center-to-center separation $R \geq 2 r$.

Figure 2. FRET efficiency as a function of acceptor number concentration $c_{A}$ per $R_{0}^{2}$ (logarithmic scale) for surface D-labeled and A-labeled spherical proteins of radius $R_{0} / 2$ (right), together, for comparison, with the equivalent trace for free $\mathrm{D}$ and $\mathrm{A}$ in the point dipole limit (left). Inset: FRET efficiencies as above $v s . c_{A}$ on linear scale, for comparison with Figure 3.

Figure 3. Optimal fits of theoretical model of FRET (lines) between surface D- and Alabeled Fab probes of GPI-anchored 5'NT partitioning between putative raft and non-raft areas of the apical cell plasma membrane of MDCK Type II cells to data (open circles) of Kenworthy and Edidin (Reference 17, Figure 4b, D-Fab: A-Fab 1:3; additional experimental data points corresponding to higher fluorescence intensities not appearing in that figure were supplied, and were included in the analysis presented). The fits were obtained using intensity calibration factors of (3, 9 and 27$) \times 10^{-6}$ per $R_{0}^{2}$ (i.e., per $25 \mathrm{~nm}^{2}$ ) [respectively, short dashed, solid, and long dashed lines] corresponding to the factor supplied by the authors for projected area [20] modified to take account of the presence of microvilli as described in the text. The apparent offset in FRET efficiency at zero concentration of acceptor accounts for the small degree of FRET to native acceptors (2.5\%) observed experimentally in absence of added specific acceptor. 


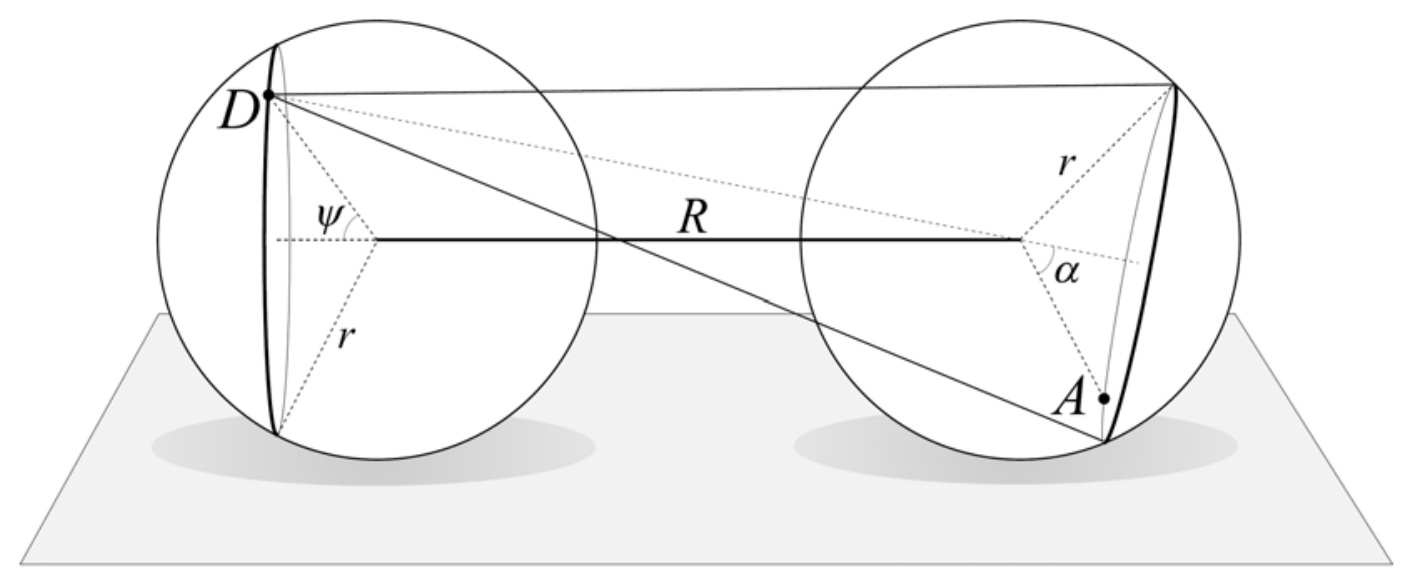

Fig 1 


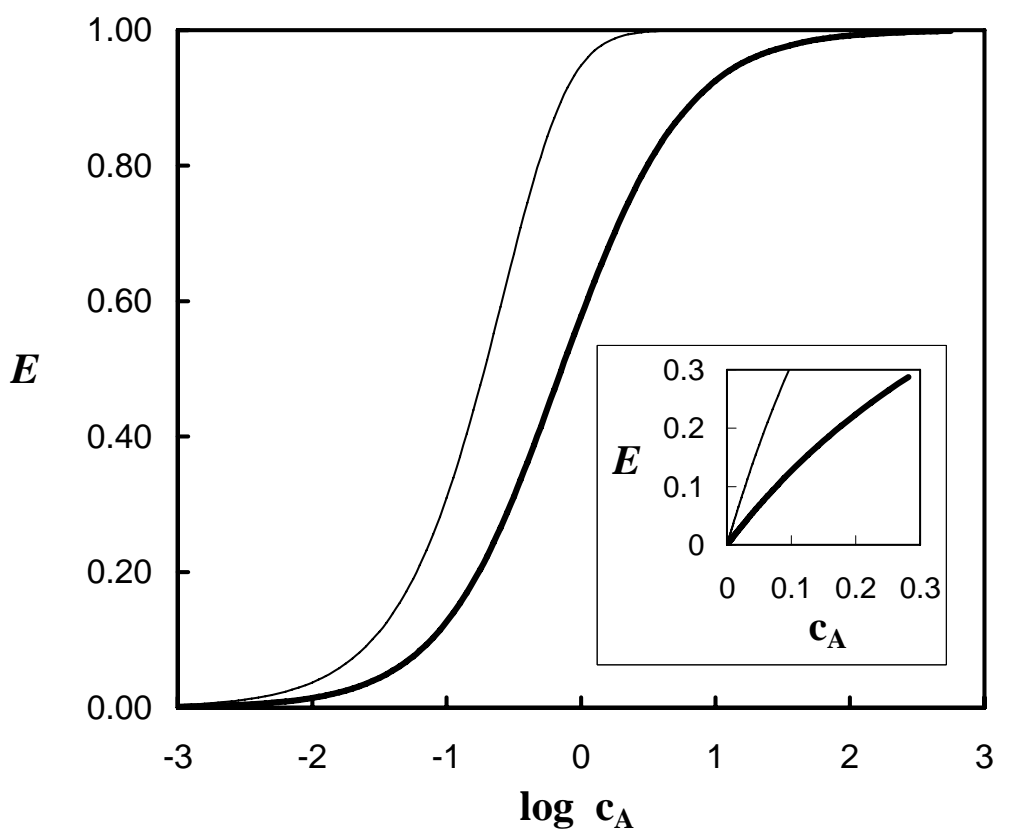

Fig. 2 


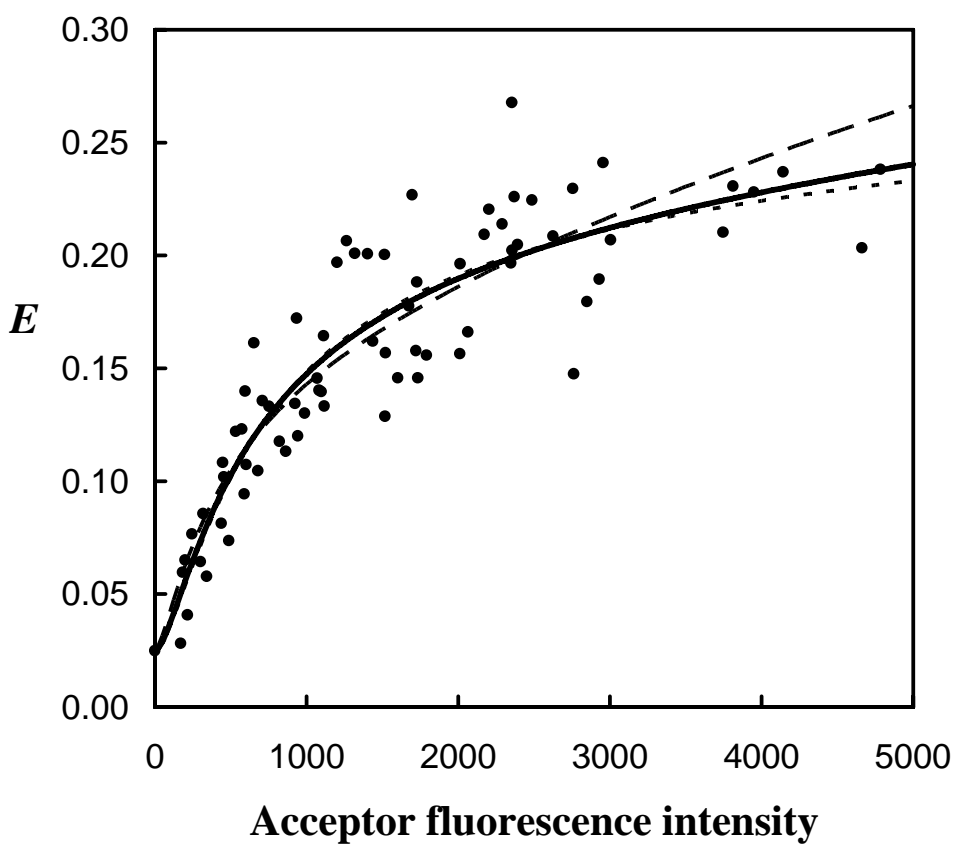

Fig .3 